\title{
The Calibration of EUSO Balloon using airborne light sources mounted to a Helicopter
}

\author{
James Adams* \\ Univ. of Alabama in Huntsville \\ E-mail: jim.adams@uah.edu
}

Malek Mustafa, Matthew Rodencal, Evgeny Kuznetsov, Jurgen Sawatzki, John Watts, Massimiliano Bonamente and Douglass Huie

Univ. of Alabama in Huntsville

\section{Mark Christl}

NASA Marshall Space Flight Center

\section{Johannes Eser and Lawrence Wiencke}

Colorado School of Mines

\section{for the JEM-EUSO Collaboration}

\begin{abstract}
The Extreme Universe Space Observatory (EUSO) Balloon was launched from Timmins, Ontario, Canada just at sunset on the moonless night of August 24, 2014. Before the balloon reached its float altitude, a helicopter carrying UV flashers and a UV laser took off. For the next 2.5 hours the helicopter circled under the balloon operating the UV flashers and a UV laser to simulate the signals from extreme energy cosmic rays. Many of these signals were recorded onboard EUSO Balloon. The laser and its use for calibrating EUSO Balloon are discussed in another paper in this conference. In this paper the helicopter operations, the flashers carried on the helicopter and the method for calibrating of EUSO Balloon using these flashers is discussed.
\end{abstract}

The 34th International Cosmic Ray Conference,

30 July- 6 August, 2015

The Hague, The Netherlands

\footnotetext{
* Speaker.
} 


\section{Introduction}

A prototype of the Extreme Universe Space Observatory (EUSO Balloon) flew on a stratospheric balloon launched from Timmins, Ontario, Canada on a moonless night in August of 2014. The objective of this flight was to test all the main subsystems of the JEM-EUSO space mission, measure the dark sky background and detect ultraviolet calibration signals (see von Ballmoos et al. in these proceedings for more details). One of the components of JEM-EUSO is the Global Light Source (GLS). In preparation for the launch of the JEM-EUSO mission, GLS units will be installed on remote high altitude sites around the world to serve as in situ calibrators for the JEM-EUSO instrument.

During the flight of EUSO Balloon, a prototype of the GLS was flown under the balloon to create artificial cosmic-ray-like signals. Many of these signals were recorded by the instrument. The recorded signals will be used to determine the absolute sensitivity of the instrument, measure the point-spread function of the focal spot and for other purposes (see Attallah and Eser in these proceedings).

In this paper we will report on the helicopter operations including the tracker system that was used to navigate the helicopter under the balloon, the design of the ultraviolet flashers that were included in the GLS and how these flashers will be used to determine the absolute sensitivity of the EUSO Balloon instrument.

\section{Pre-flight Preparations}

\subsection{Design of the Global Light System}

The GLS is described in Wiencke et al. (2013). All the GLSs will contain flashers and half of them will also contain a laser. A prototype of the GLS flown under the balloon consisted of a laser (see Eser et al. in these proceedings) and two kinds of flashers, one is based on a Xe flash lamp and the other uses a UV-emitting LED. Figure 1 shows the flashers mounted in the enclosure used during the helicopter flights.

The Xe flash lamp (see figure 1) is a Hamamatsu Xenon lamp \#6604. This lamp (chosen for the GLS system) is typically used as an aircraft warning light on towers. The angular distribution of the light from the flasher was reported by Adams et al. (2012) and is shown in figure 4 of that paper. From this figure one can see that the emission is uniform to $<5 \%$ within $\pm 30^{\circ}$ of the optical axis of the lamp. This is a larger than the angular range of rays going from the helicopter to EUSO Balloon. The flash from this lamp (as received from Hamamatsu) is too short so the circuitry controlling the lamp was modified to stretch the light pulse to $20 \mu \mathrm{s}$. This lamp emits light over a broad spectrum between 240 and $2000 \mathrm{~nm}$. The prototype unit was fitted with a band pass filter at $355 \mathrm{~nm}$ with a $10 \mathrm{~nm}$ bandwidth.

The prototype electronic control system for this lamp was designed to operate the lamp at $500 \mathrm{~V}, 600 \mathrm{~V}, 800 \mathrm{~V}$ or $1000 \mathrm{~V}$ to provide more dynamic range in order to be sure that, at some voltage, the flashes could be seen but woulld not saturate photon counting system in EUSO Balloon.

The UV-emitting diode used in the prototype GLS (see figure 1) is a Engin Inc. type LZ4$00 \mathrm{U} 600^{1}$ with a maximum optical power of $1 \mathrm{~W}$. The emission from this LED has a narrow spec-

\footnotetext{
${ }^{1}$ http://www.ledengin.com/files/products/LZ4/LZ4-00U600.pdf
} 


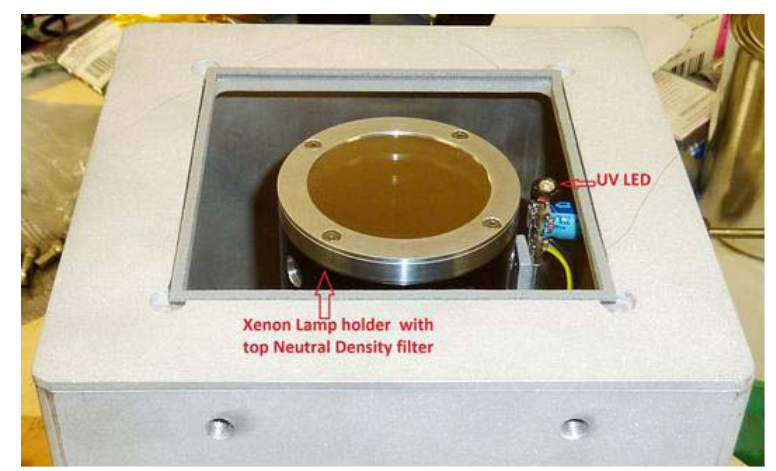

Figure 1: The Xe and LED flashers shown in their flight enclosure.

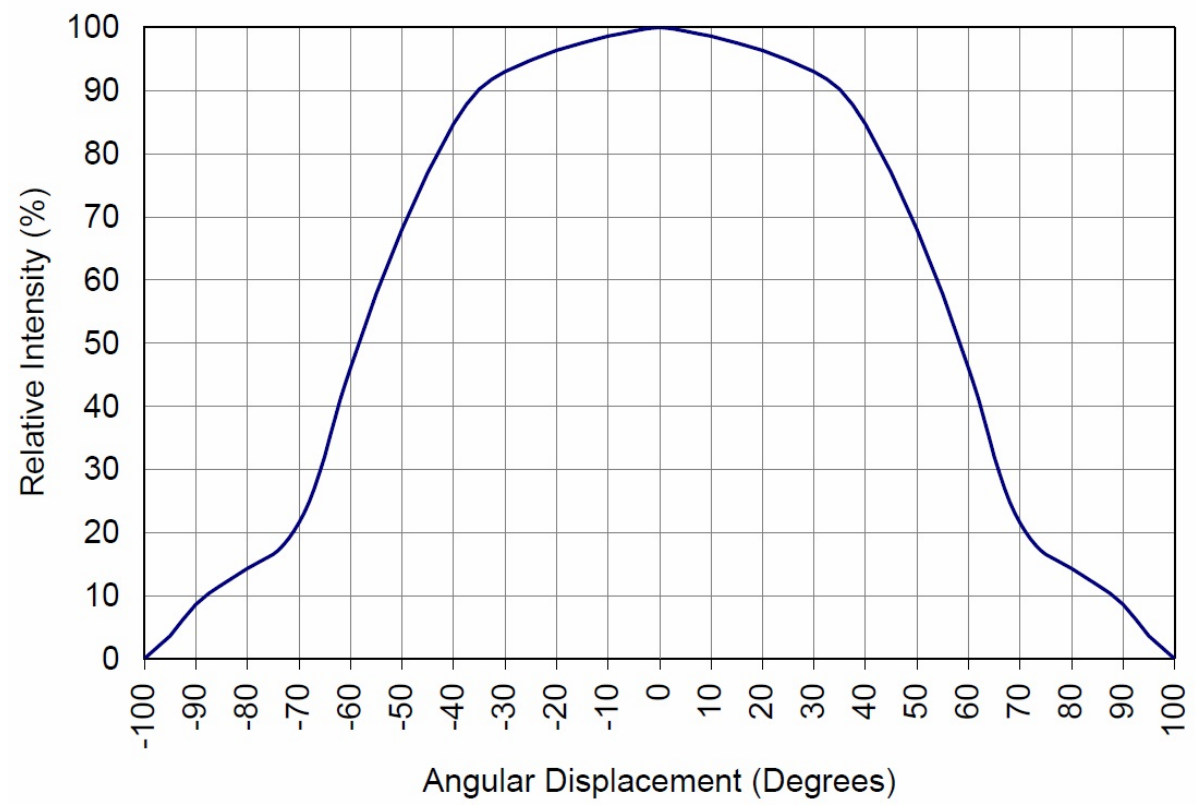

Figure 2: The relative intensity of the LED as a function of field angle.

trum that peaks at $365 \mathrm{~nm}$. The angular distribution of the emission (shown in figure 2) varies by $<7 \%$ out to $\pm 30^{\circ}$ from the normal which is more than sufficient for this application. The control electronics for the LED increased its operating voltage from 2.7 to 4.3 volts in 12 steps, each one GTU (or $2.5 \mu \mathrm{s}$ ) in length. LED driver board boosts the control voltage by a factor of five to achieve maximum optical output power from the LED. Each LED flash lasted $30 \mu$ s or 12 GTUs. With each successive GTU the intensity of the LED was increased as shown in figure 5.

\subsection{Design and testing of the balloon tracker system}

In order to fly the helicopter within the field of view of EUSO Balloon special navigation equipment was required. Utilizing experience gained from the BALLOONSAT project of the student Space Hardware Club at the University of Alabama in Huntsville ${ }^{2}$. A tracker beacon con-

\footnotetext{
${ }^{2} \mathrm{http} / / /$ space.uah.edu/
} 


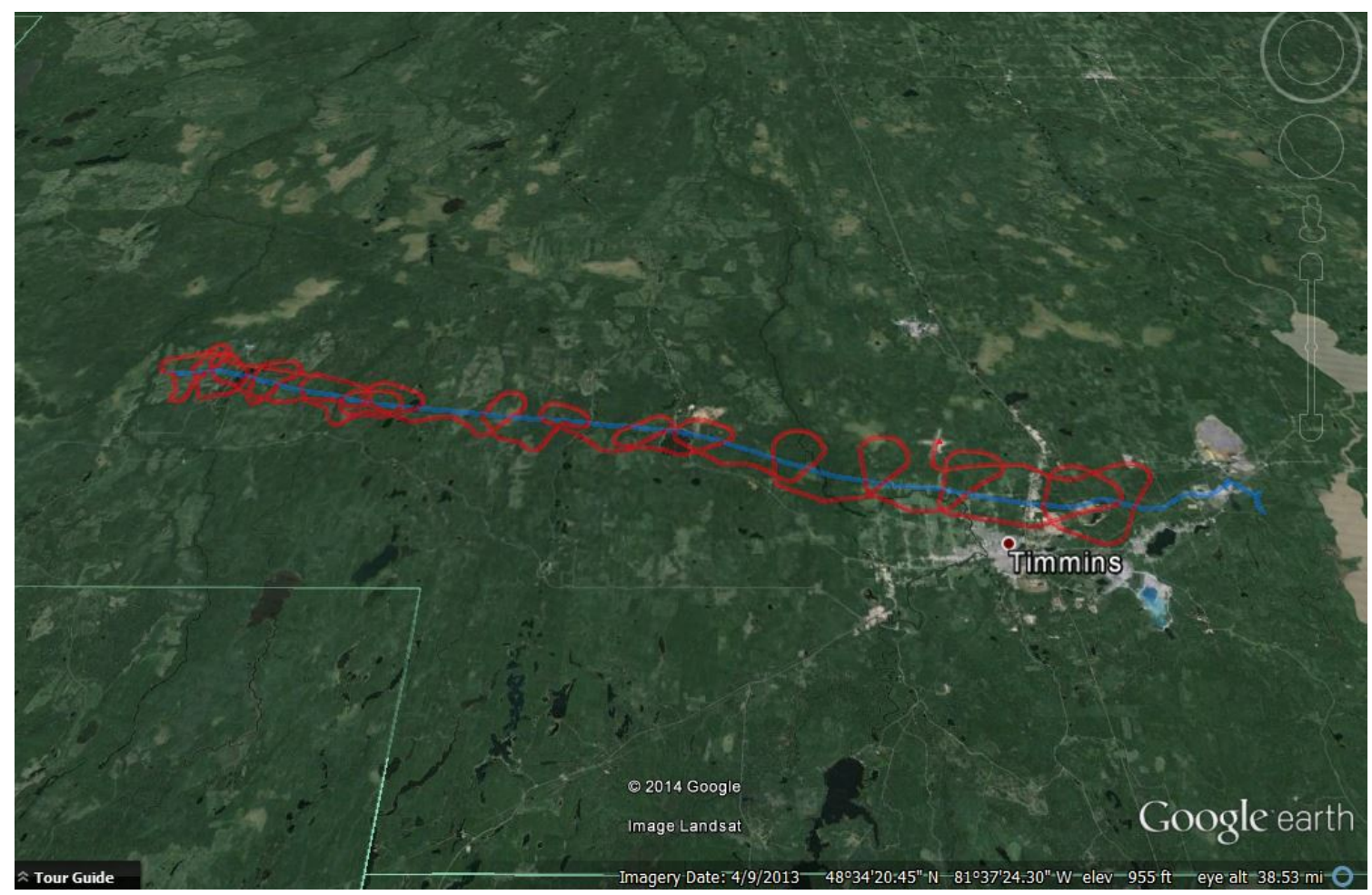

Figure 3: The path in blue is the track of EUSO Balloon from the time it launched until the helicopter under-flight ended. Shown in red is track of the helicopter.

sisting of a GPS receiver coupled with a HAM transmitter was developed. Two of these beacons were flown on EUSO Balloon. The signals from the beacons were received using a Kenwood software-defined radio ${ }^{3}$. This radio contains a second GPS receiver. It is capable of decoding the GPS signals from the beacons and displaying the direction and range to a point directly below the balloon at the altitude of the radio. To facilitate communications with the helicopter pilots, the radio was connected to a laptop computer which was programmed to enable the positions of both the balloon and the helicopter to be displayed on a Google Earth.

\subsection{Flight Tests}

A series of helicopter test flights were conducted to test the laser, the flashers and the tracking system. The first two flights were arranged by the Rotocraft Center ${ }^{4}$ at UAH and launched from the Tullahoma Regional Airport. During these flights $1500 \mathrm{~g}$ latex balloons carrying the tracker beacons were launched by members of the UAH Space Hardware Club. These balloons were chased by a helicopter carrying two of our team members to operate the GLS and to assist the pilot in navigating the helicopter under the balloon.

The helicopter for the flight operations was a Bell 205 provided by Helicopter Transport Services Canada ${ }^{5}$ based in Carp, Ontario, Canada. The flasher system was mounted in a basket at-

\footnotetext{
${ }^{3}$ Kenwood model 144/430 MHz FM Dual Bander TH-D7E

${ }^{4}$ http://www.uah.edu/rsesc

${ }^{5}$ http://www.htsc.ca/
} 


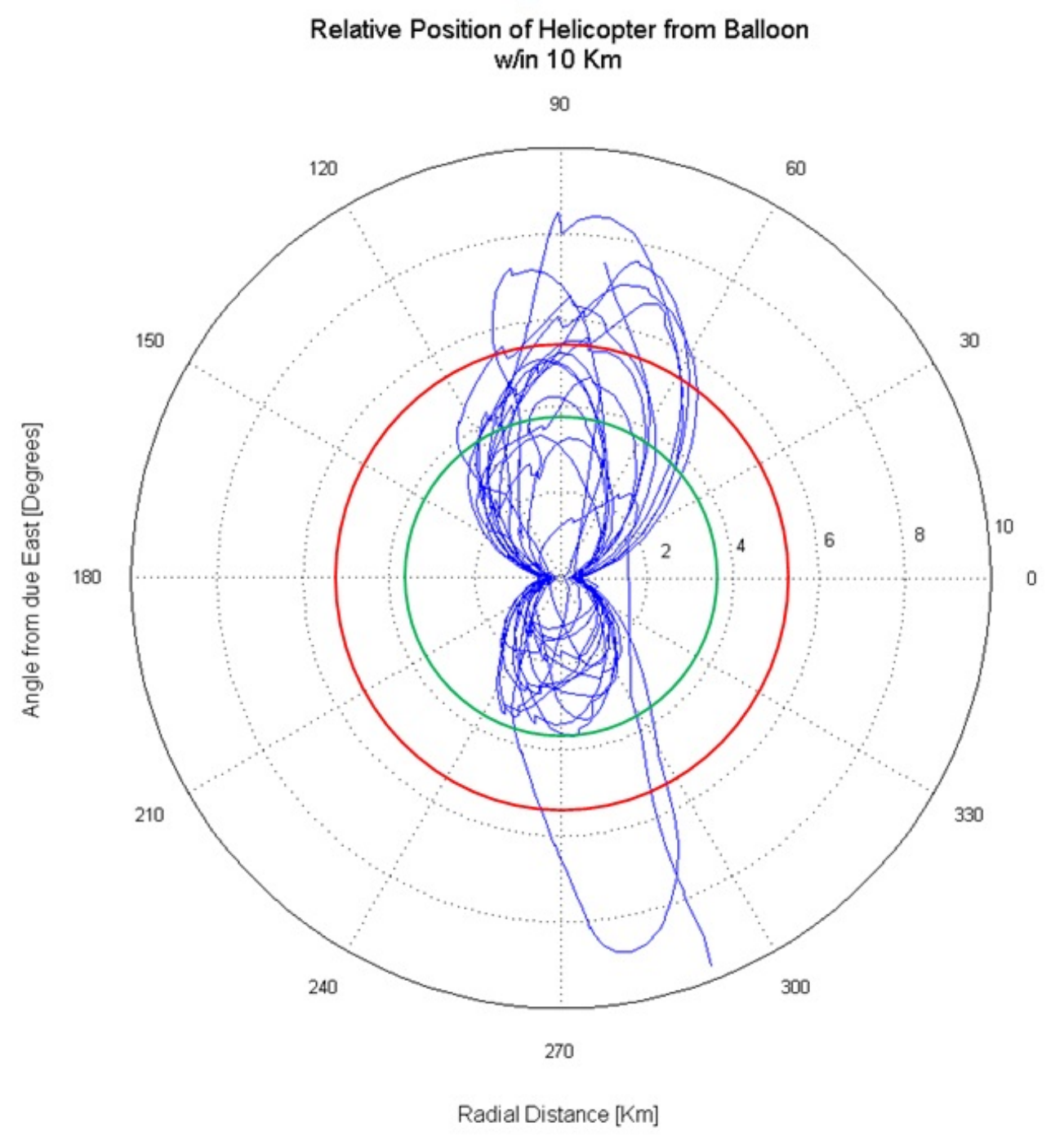

Figure 4: The helicopter's position relative to a point directly under the balloon at the altitude of the helicopter is shown in blue. the green and red circles are inscribe and circumscribe EUSO Balloon's field of view.

tached to the side of the helicopter and the laser was on the floor in front of the back seat and aimed out the side door above the basket. Two of our students, Matthew Rodencal and Johannes Eser, were in the back seat. Matthew provided the navigational guidance to the pilots using the tracker system he had developed while the laser and flashers were operated by Johannes Eser. We conducted two test flights around Carp to test our equipment and to familiarize the pilots with the flight operations.

\section{Flight Operations}

EUSO Balloon was launched from the Timmins Stratospheric Balloon Base ${ }^{6}$ in Timmins,

\footnotetext{
${ }^{6} \mathrm{http}: / /$ stratocat.com.ar/bases/80e.htm
} 


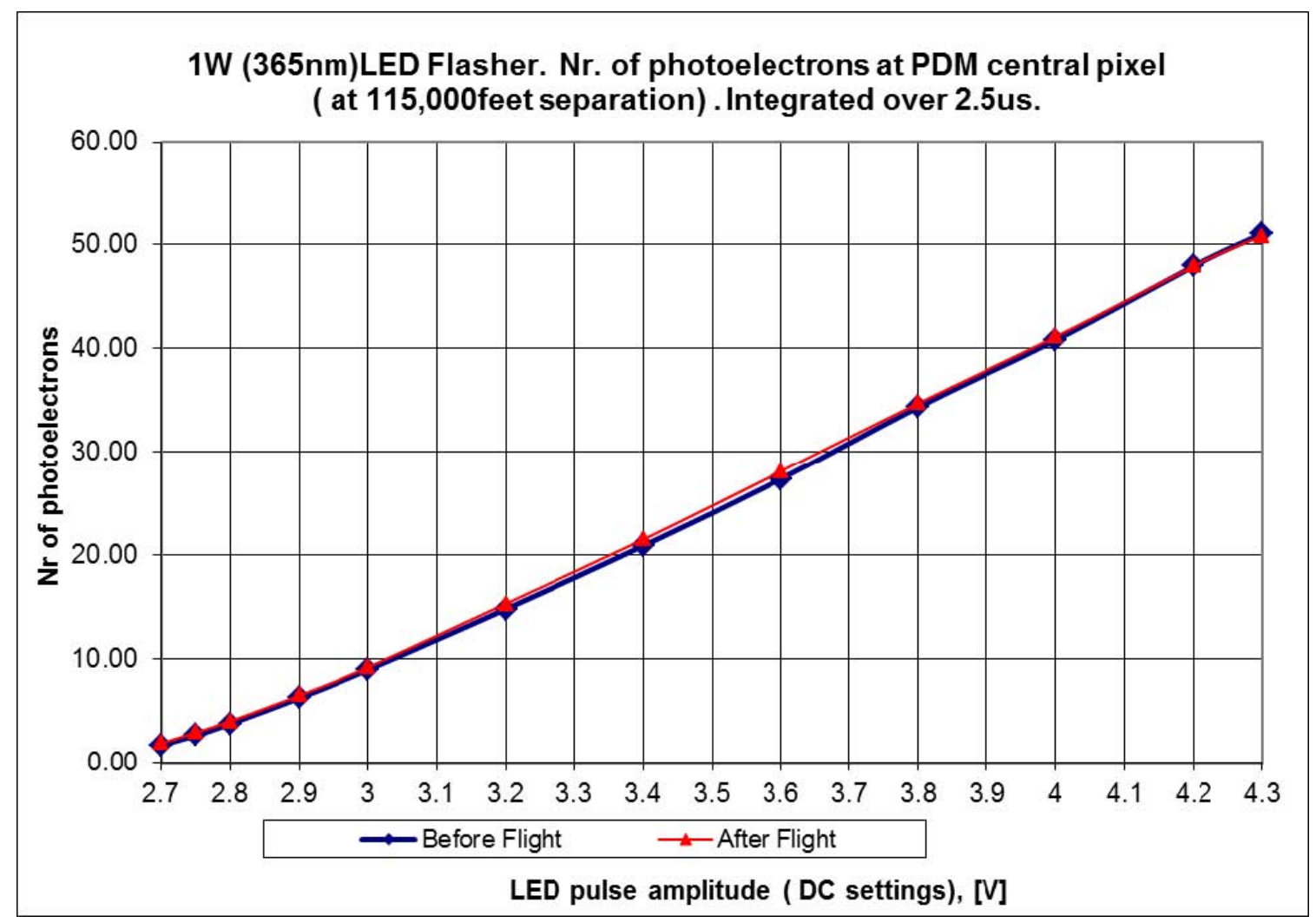

Figure 5: Comparison of pre- and post-flight calibration of the LED flasher. The output of the LED was increased in 12 steps during each flash. Each step lasted 1 GTU. The intenstites at these steps are shown by the diamond symbols in this figure.

Ontario, Canada at sunset on August 24, 2014, local time. As the balloon was ascending, the helicopter arrived from Carp and refueled. Approximately two hours after the balloon was launched the helicopter took off and gave chase. After arriving under the balloon, flasher and laser operations began. The firing sequence was that the LED flasher fired first for $30 \mu \mathrm{s}$. The laser was fired $95 \mu \mathrm{s}$ after the LED fired. The Xe flasher fired $10 \mu \mathrm{s}$ after the laser fired and it lasted $20 \mu \mathrm{s}$. The whole sequence lasted $\sim 125 \mu \mathrm{s}$. The helicopter circled under the balloon while operating the laser and the flashers for $\sim 2.5$ hours. Figure 3 shows in blue the track of the balloon from launch until the helicopter under-flight ended. Shown in red, in the same figure, is the trajectory of the helicopter as it circled under the balloon.

The GPS positions of the balloon and the helicopter were recorded and used to determine the position of the helicopter relative to the point directly below the balloon. Figure 4 shows the path of the helicopter relative to the location of the EUSO Balloon. As can be seen, the helicopter was mostly within the field of view of EUSO Balloon.

The sequence of LED flasher-Laser-Xe flasher was repeated at a cadence of $19 \mathrm{~Hz}$, but these operations could not be synchronized with the operation of EUSO Balloon which captured 128 GTU video clips at a cadence of $20 \mathrm{~Hz}$. Even though the duty cycle of the GLS was 0.00295 and that of EUSO Balloon was 0.00256, we observed over 100 chance coincidences when EUSO Balloon captured the signals from one or both of the flashers. Because the clocks on the helicopter 
and the balloon are not sychronized, The signal captured in a GTU on the helicopter may consist of light from part of two successive GTUs on the helicopter.

During post-flight calibration we found that the bright light from the Xe flasher had damaged the UV filter we used. We have measured the rate of degradation in this filter to estimate the intensity of the Xe flashes during the flight. Pre- and post-flight calibration of the LED flasher are shown in figure 5. These data show that the LED flashes did not degrade during the flight.

\section{Data Analysis}

By searching the packets of images for ones with photoelectron counts above background we were able to locate over 98 that contained LED flasher data and 142 that contained Xe flashes. The role of the flashers is to provide light sources of known intrinsic luminosity that can be used to determine the sensitivity of the EUSO Balloon instrument to the signals from extensive air showers. To date these light sources have been calibrated only against a Hamamatsu type R890003 phototube (the same generic type as used in EUSO Balloon). This phototube is equipped with a BG3 optical filter(the same as are those used in EUSO Balloon).

In table 1 we present data from 3 LED flashes that occurred under clear sky conditions as determined from the onboard IR camera (see Rodriguez et al. in this conference). The focal spots we mostly contained in a $5 \mathrm{X} 5$ pixel area centered on the brightest pixel. The location of the center of the focal spot from each flash is given in columns 2 and 3 in units of pixels on the PDM (which is $48 \mathrm{X} 48$ pixels). For each flash, we measured the signal in those GTUs for which the integrated photoelectron count over the $5 \mathrm{X} 5$ pixel area was clearly above background. The background was deteremined by averaging the signal over 10 GTUs following the end of each flash. The number of GTUs that the signal was above background is given in column 4. Because the LED intensity was increased with each successive GTU during a flash, these are the last GTUs in the flash. The total signal collected summed over all measurable GTUs in each flash is given column 5. Column 6 gives the calculated photon fluence striking the entrance aperture during the measureable GTUs of each. These values were calculated taking into account the distance between the helicopter and the balloon and the Rayleigh scattering loss, using the formulas in Dawson (2006). The principal source of uncertainty in the calculated photon flux is calibration of the LED. As can be seen in Figure 3 of Moretto et al. (2015), the sensitivity of the phototubes used in EUSO Balloon vary by a factor of $\sim 2$. So we should expect that the sensitivity of phototube used to calibrate the LED could differ from those at the focal spots of the flashes in EUSO Balloon by a similar factor, making the calculated photon fluence in column 6 uncertian by a factor of $\sim 2$.

\section{Conclusions}

The results obtained with the flasher system demonstrate that the sensitivity of JEM-EUSO to light flashes deep in the atmosphere can be measured using a calibrated flasher system. We plan to calibrate the flashers against a Si photodiode with a U.S. Natilonal Institute of Standards and Technology (NIST) traceable calibration. This will allow us to determine the absolute intrinsic 


$\begin{array}{cccccc}\begin{array}{c}\text { Time } \\ \text { (hh:mm:ss) }\end{array} & \begin{array}{c}\text { X } \\ \text { (pixels) }\end{array} & \begin{array}{c}\text { Y } \\ \text { (pixels) }\end{array} & \begin{array}{c}\text { Duration } \\ \text { (GTUs) }\end{array} & \begin{array}{c}\text { Signal } \\ \text { (p.e.s) }\end{array} & \begin{array}{c}\text { Signal } \\ \text { (photons) }\end{array} \\ \text { 05:31:54 } & 19 & 18 & 8 & 640 & 2.2 \times 10^{3} \\ \text { 05:39:51 } & 15 & 20 & 9 & 478 & 2.2 \times 10^{3} \\ \text { 05:41:52 } & 30 & 13 & 10 & 578 & 2.2 \times 10^{3}\end{array}$

Table 1: The data from three LED flashes observed by EUSO Balloon. The first column gives the time (UTC) that the flash occurred. The second give the location of the center of the focal spot for each flash in the PDM in units of pixels. The PDM is 48 by 48 pixels. The LED flasher operated for 12 GTUs. Each GTU its intensity increased. Column 4 tells how many GTUs at the end of the flash were bright enough to be measureable. The fifth column gives the total count of photoelectrons measured from each flash and the last column gives an estimate of the total number of photons from each flash that fell on the entrance aperture of EUSO Ballooon.

luminosity of these flashers and hence the absolute sentitivity of EUSO Balloon at these and other points on the focal surface where flasher images appear.

Acknowledgment: The corresponding authors wish to thank the EUSO IR Camera team from Spain for checking the cloud cover during the 3 flashes used in this work. The authors also wish to thank CNES and CSA for conducting the balloon flight operations. This work was supported by NASA Grant NNX 13AH53G andSpanish grants AYA2009-06037-E/AYA, AYA-ESP 2010-19082, AYA2011-29489-C03-01, AYA2011-29489-C03-02, AYA-ESP2012-39115-C03-01, AYA-ESP2012-39115-C03-03, AYA-ESP 2013-47816-C4, MINECO/FEDER-UNAH13-4E-2741,CSD2009-00064 (Consolider MULTIDARK), S2009/ESP-1496 \& S2013/ICE-2822.

\section{References}

[1] J.H. Adams et al., "'Ground Truth' calibration for the JEM-EUSO Mission", 2012 COSPAR Conference, http://arxiv.org/ftp/arxiv/papers/1302/1302.5863.pdf (2012).

[2] Bruce Dawson, "New Rayleigh Scattering Implementation in the Offline", Prodeedings of the GAP Conf. (2006).

[3] J. Eser et al., Proc. 34th ICRC, 2015, paper\# 638, "Laser reconstruction in EUSO-Balloon experiment".

[4] C. Moretto et al., Proc. 34th ICRC, 2015, paper\# 655, "Absolute calibration of the Photo-Detector Module of the EUSO-Balloon experiment and improvements for future missions".

[5] M. D. Rodriguez Frias et al., Proc. 34th ICRC, 2015, paper\# 680, "The atmospheric science of JEM-EUSO”.

[6] P. von Ballmoos, et al., Proc. 34th ICRC, 2015, paper\# 322, ”General overview of EUSO-Balloon mission".

[7] L. Wiencke et al., "The JEM-EUSO Global Light System", in the Proc. 33rd ICRC (Rio de Janeiro), http://arxiv.org/pdf/1307.7071.pdf\#page=87 (2013). 